Case Report

\title{
Management of Oral Lesions in Paraquat Poisoning - A Case Report
}

\author{
Muralee M ohan C. ${ }^{1}$, Pratiksha Shetty ${ }^{2}$, Saurabh M. Gohil ${ }^{3}$, Jitesh Sharma ${ }^{4}$, Satadru Roy ${ }^{5}$ \\ ${ }^{1}$ Professor, ${ }^{2}$ Assistant Professor , ${ }^{3,4,5}$ Post Graduate Student, Department of Oral \& Maxillofacial Surgery, A.B. Shetty \\ Memorial Institute of Dental Sciences, M angalore, Karnataka, India.
}

\begin{abstract}
*Corresponding Author : Saurabh M. Gohil, Post Graduate Student, Department of Oral \& M axillofacial Surgery, A.B. Shetty M emorial Institute of Dental Sciences, Nitte University, Mangalore - 575018, Karnataka, India, Phone - 9845197263/09819929726, E-mail : dr.saurabhgohil@gmail.com.

Received

:20.07.2016

Review Completed : 12.01.2017

Accepted

:02.02.2017

Keywords : paraquat, poisoning, choline salicylate, benzalkonium chloride

Abstract

Paraquat is a herbicide with a good occupational safety record, but a high mortality after intentional ingestion that has proved refractory to treatment. For nearly three decades paraquat concentration-time data have been used to predict the outcome following ingestion, however, none of the published methods has been independently or prospectively validated. However, paraquat poisoning is an uncommon entity in India. We report a case of oral lesions associated with paraquat poisoning and management of the mucosal burns associated with it along with medical management of paraquat poisoning in general.
\end{abstract}

\begin{tabular}{|c|}
\hline Access this article online \\
\hline Quick Response Code \\
\hline
\end{tabular}

\section{Introduction}

Poisoning by pesticides and other agricultural chemicals is a major public health issue especially in the developing countries. There are about 20,000 annual fatalities and more than two million hospitalizations due to poisoning by pesticides and other agricultural chemicals (1). Paraquat (1,1'-dimethyl-4,4'-bipyridinium) dichloride is a nonselective contact herbicide that has been widely used in many countries since the 1960s. It is fast-acting, rain-fast and facilitates 'no-till' farming, but it has attracted controversy because of a high mortality in cases of self harm (typical case fatality 50-90\%) (2). Paraquat, a widelyused herbicide, remains a major cause of death in developing countries. In fact, more than 200 deaths were reported in the first two decades after its widespread use began in 1958. Although freely available as a liquid concentrate $(29.1 \%)$ in the Indian market for agricultural use, it is an uncommon pesticide in India (3).
Paraquat is highly toxic and causes damage to the lungs, liver and kidneys. Paraquat poisoning can be classified into three categories, namely: mild poisoning (20 mg paraquat ion per kilogram of body weight) in which the patients generally have minor gastrointestinal symptoms but usually fully recover; severe poisoning (20-40mg paraquat ion per kilogram of body weight) in which the patients develop acute renal failure, acute lung injury and progressive pulmonary fibrosis with death occurring in two to three weeks from respiratory failure; and fulminant poisoning (40 mg paraquat ion per kilogram of body weight) in which the patients develop multiple organ failure leading to death within hours or 1-4 days due to circulatory failure (4). Ingestion of smaller amounts primarily results in progressive pulmonary damage secondary to diffuse alveolar damage with resultant acute respiratory distress syndrome (5). Therefore, chemical burns in oral and gastrointestinal mucosa are generally involved. 
The cytotoxic effects of paraquat have been attributed to the generation of superoxide radicals after reduction of paraquat by intracellular oxidases and amplified generation of further reactive oxygen species results in profound pulmonary injury (6).

\section{Case Report}

Hereby, we present a case of paraquat poisoning which was referred to us from the Department of General Medicine of our Hospital. Patient came with a chief complaint of accidental swallowing of pesticide (paraquat) while opening the bottle (Patient was unaware about the pesticide in the bottle). The patient experienced nausea and vomiting 1-2 hours after accidental ingestion of the pesticide and was brought to the hospital by the relatives within an hour since the symptoms appeared. In the emergency room the patient reported sore throat and epigastric pain, but denied any shortness of breath or any difficulties in breathing. There were no underlying medical problems. Physical examination in the emergency room revealed an alert, fully conscious patient in no acute distress, with normal vital signs. A chest radiograph was clear, without definite infiltrates, and the cardiac contour was normal. Electrocardiogram revealed sinus tachycardia. Intravenous fluids were started and the patient was admitted to the medical intensive care unit for close observation and further evaluation. Other supportive treatments included intravenous fluids and analgesics to control epigastric pain.

Our patient did not have any systemic complications as repeated Liver Function tests and Renal Function tests were within normal range. However, patient developed severe erythematous lesions covered with slough throughout the oral cavity including floor of the mouth, ventral surface of tongue and palate (Figure 1).

There was severe pain and burning sensation associated with dysphagia and excess salivation. There were also lesions present on the right cheek of the patient. Our treatment protocol included daily thorough debridement of slough followed by application of combination of Choline salicylate and Bezalkonium Chloride gel along with

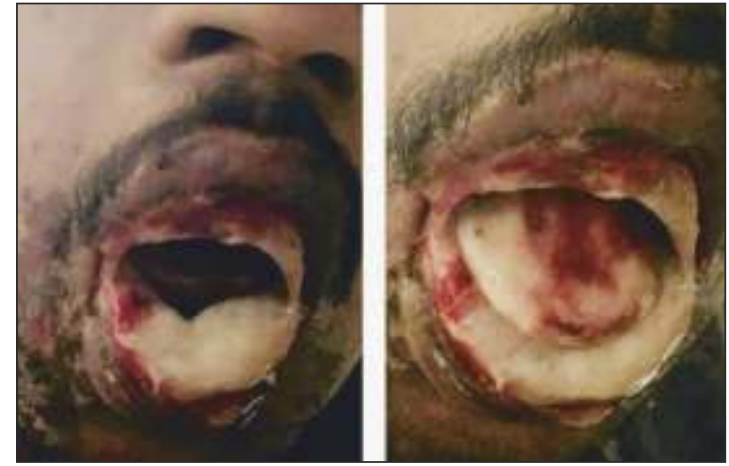

Fig. 1 : Initial presentation showing severe sloughing and erythematous changes of oral mucosa

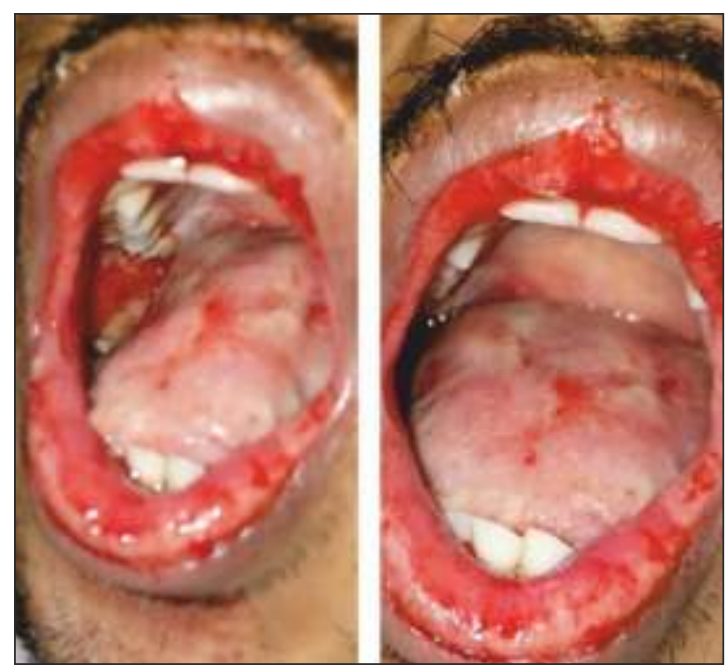

Fig. 2 : Appearance of oral mucosa following slough debridement
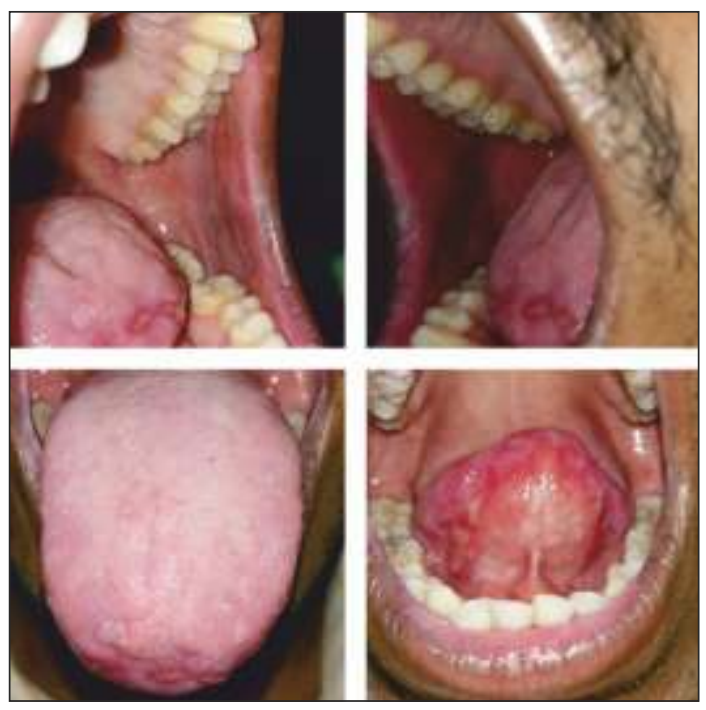

Fig. 3: No sloughing and few patches of erythema after 15 days.

Lignocaine gel and topical application of contents of Multivitamin capsules. 
Patient had severe pain and burning sensation even after topical application. Debridement of slough after topical anaesthetic gel application was done until fresh bleeding was seen from the tissues (Figure 2).

Daily debridement of slough for 3-4 days was done because there was recurrence of slough formation. But after 5 days of regular debridement and topical application, the patient showed signs of improvement with less sloughing of tissues and decreased salivation. After 1 week, the patient was much comfortable symptomatically and on discharge, there was minimal sloughing and areas of erythematous lesions. Patient was advised topical application of choline salicylate and benzalkonium chloride thrice a day on discharge.

On follow-up after 15 days, patient's condition improved significantly and there were no signs of sloughing, but very few patches of erythema were still present (Figure 3).

\section{Discussion}

Poisoning from pesticides and other agricultural chemicals is a major public health problem worldwide, in both developing and developed communities. Globally, there are about 20,000 annual fatalities and more than 2 million hospitalizations due to poisoning by pesticides and agricultural chemicals. (7) Severe paraquat poisoning is characterized by multiple-organ failure, involving mainly the lungs, kidneys, liver, myocardium, and adrenal cortex. The lung is a major target organ in paraquat poisoning, and respiratory failure from acute pulmonary fibrosis is the most common cause of death. The clinical manifestations range from local irritation to multiple-organ failure and death. Paraquat (especially concentrated paraquat) is corrosive and can severely injure skin, eyes, and gastrointestinal mucosa. Systemic manifestations depend on the amount of paraquat ingested. (4) Although we did not confirm the presence of paraquat by the urinary dithionite test and plasma paraquat levels, the clinical history and presentation, and documentation of paraquat made the diagnosiscertain.

M edical management of paraquat self-poisoning (8) -

\section{Resuscitation}

Patients in extremis have no realistic hope of recovery with current treatments. Treatment of such patients should be palliative once the diagnosis is established. Otherwise, the standard principles of resuscitation (assessment and management of airway, breathing and circulation) should generally be followed as per routine guidelines. The airway may be compromised due to mucosal toxicity or the presence of vomitus. Tachypnoea and/or hypoxia may be due to metabolic acidosis, aspiration and/or acute alveolitis and a blood gas and chest radiograph may help make the correct diagnosis.

\section{Confirming the diagnosis and risk assessment}

A semi-quantitative test using bicarbonate and sodium dithionite can be used as a bedside test to confirm systemic paraquat toxicity. In an alkaline medium, sodium dithionite reduces paraquat to a blue radical. If the urine paraquat concentration is more than $1 \mathrm{mg} / \mathrm{l}$, the urine will appear blue and this finding alone indicates a very poor prognosis. Measurement of plasma paraquat concentration is also useful both to confirm poisoning and predict prognosis.

\section{Investigations}

In addition to the paraquat concentrations discussed above, biochemistry (electrolytes and renal and liver function tests), and haematology (full blood count) should be done at least daily. A chest radiograph should be performed if pneumomediastinum, pneumothorax or lung fibrosis is suspected. A CT scan of the chest may be useful in detecting early lung fibrosis or assessing long-term damage in survivors.

\section{Clinical monitoringand ongoing care}

Patients should be monitored for the development of:

a. Acute renal failure - Daily fluid balance should be maintained with the aim of ensuring a good urine output without overloading the patient.

b. Liver toxicity - Clinical examination will usually detect jaundice and right hypochondrial pain.

c. Respiratory failure - respiratory rate, auscultation of the lungs (for crepitations) and measurement of peripheral oxygen saturation should be performed on at least a 
twice-daily basis.

d. Mucosal injury - patients develop severe oral ulcers within a few days after ingestion of paraquat. This generally prevents patients from taking adequate food or oral fluids for up to 10 days. Early insertion of a nasogastric feeding tube will ensure adequate nutrition.

5. Elimination enhancement: haemodialysis(HD)/ haemoperfusion (HP)

Haemodialysis and Haemoperfusiom are part of the standard treatment in many centers. However, the kinetics of paraquat suggest the benefits of this will be very limited for two reasons. Firstly, the endogenous clearance is high in the first 6 to 12 hrs and thus most paraquat is eliminated rapidly anyway and the additional amount eliminated will be relatively modest. Secondly, the time frame in which the increased elimination will have an impact on the distribution into lungs is very short.

M anagement of paraquat poisoning has remained mostly supportive and the results of treatment for paraquat poisoning, including absorbents, pharmacological approaches, radiotherapy, haemodialysis and haemoperfusion were disappointing. Currently, there are no true pharmacological antagonists for paraquat and there are no chelating agents capable of binding the poison in the blood or other tissues (8).

However, our patient had only oral mucosal lesions, few cutaneous lesions and seems to have no systemic

\section{References}

1. Patterns and problems of deliberate self-poisoning in the developing world. QJM 2000; 93:715-31. M, Eddleston.

2. Paraquat. In: Handbook of Pesticide Toxicology, 2nd edn. San Diego, Academic Press, 2001:1559-603. LockEA, Wilks M F.

3. Fatal paraquat poisoning: report of two cases. J Assoc Physicians India 1999; 47:831-2. Singh S, Bambery P, Chaudhry D, et al.

4. Paraquat poisoning. Respir Care 2005; 50:383-5. C., Sittipunt.

5. Paraquat poisoning. An overview of the current status. Drug Saf 1990; 5:243-51. Bismuth C, Garnier R, Band FJ, M uszynski J, Keyes C.

6. Cyclophosphamide treatment of paraquat poisoning. Thorax 1996; 51:659-60. Comment on: Thorax 1996; 51:661-3. CG., Newstead. complications. Since our patient was admitted in General Medicine department, regular liver finction tests, renal function tests, lung function and chest x-ray were done to ascertain that no systemic complication are there. There are negligible reports regarding oral lesions in paraquat poisoning and none related to the management of oral lesions. Our management protocol gave satisfactory results, therefore, can be considered for routine management of oral lesions in paraquat poisoning. Generation of highly reactive oxygen and nitrite species results in toxicity in most organs and tissues of the body. (8) Since oral mucosa is subjected to chemical burns invariably during oral consumption, management of this tissue becomes essential part of paraquat poisoning patients. We suggest daily debridement of slough and topical application of choline salicylate, benzalkonium chloride and contents of Multivitamin capsules till healthy tissue formation is seen. This is so as to encourage tissue regeneration and healing. Since

\section{Conclusion}

A mucosal burn due to chemical insults is of common occurrence but often neglected due to more pressing medical complications associated with it. In this article we present simultaneous management of mucosal burns along with systemic monitoring and treatment, so as to help in early oral nourishment, helping to early recovery of the patient. Therefore we propose early involvement of maxillofacial surgeons in such cases to help early recovery and avoid extended duration of discomfort to the patient.

7. Pesticides and the third world. J Toxicol Environ Health 1991;32(1):11-31. G., Forget.

8. Medical management ofparaquat ingestion. $\mathrm{Br} J$ Clin Pharmacol 72:5;745-757. Gawarammana IB, Buckley NA.

9. M anagement of Poisonings: Core Curriculum 2010. American Journal of Kidney Diseases, Vol 56, No 4 (October), 2010: pp 788-800. Winchester JF, Harbord NB, Rosen H.

10. Experience with paraquat poisoning in a respiratory intensive care unit in North India. Singapore Med J 2006; 47(12) : 1033. Agarwal R, Srinivas R, Aggarwal A N, Gupta D. 